\title{
Comparison of Reverse Total Shoulder Arthroplasty vs Hemiarthroplasty for Acute Fractures of the Proximal Humerus: Systematic Review
}

\author{
${ }^{1}$ Amrut U Borade, ${ }^{2}$ Filippo Familiari, ${ }^{3}$ Kyubo Choi, ${ }^{4}$ Jacob Joseph, ${ }^{5}$ Edward G McFarland
}

\begin{abstract}
The optimal treatment of proximal humerus fractures is debated; however, reverse total shoulder arthroplasty (RTSA) has become increasingly popular as the primary treatment. We systematically reviewed the PubMed, EMBASE, and Scopus databases to identify English-language clinical studies (evidence levels I through IV) comparing the results of RTSA with those of hemiarthroplasty $(\mathrm{HA})$ for the treatment of acute proximal humerus fractures. We evaluated the following outcomes: Range of motion, patient-reported outcome measures (including pain relief), and complications. We identified eight published studies comparing RTSA with HA for treatment of acute proximal humerus fractures. The RTSA group (180 patients) showed significantly better postoperative pain relief, active anterior elevation, Constant-Murley scores, and American Shoulder and Elbow Surgeons (ASES) scores compared with the HA group (439 patients; all $p<0.05$ ). There were no significant differences between the two groups for overall complications (RTSA, $11.8 \%$ vs HA, $20.8 \%$ ), infections (2\% for both groups), dislocations (RTSA, $0 \%$ vs HA, $2.5 \%$ ), or nerve injury (RTSA, $1 \%$ vs HA, $2.8 \%$ ). Scapular notching occurred in $15.8 \%$ of RTSA cases (grade I, 53\%; grade II, 32\%; grade III, 16\%; and grade IV, $0 \%$ ). In the treatment of acute proximal humerus fractures, RTSA provided better pain relief, postoperative anterior elevation, and outcome scores after surgery compared with $\mathrm{HA}$ and had similar complication rates.
\end{abstract}

Keywords: Complications, Outcomes, Pain, Reverse total shoulder, Shoulder hemiarthroplasty, Surgery, Systematic review.

How to cite this article: Borade AU, Familiari F, Choi K, Joseph J, McFarland EG. Comparison of Reverse Total Shoulder Arthroplasty vs Hemiarthroplasty for Acute Fractures of the Proximal Humerus: Systematic Review. J Postgrad Med Edu Res 2017;51(4):182-187.

Source of support: Nil

Conflict of interest: None

\section{INTRODUCTION}

Proximal humerus fractures are the third most common extremity fracture in patients older than 60 years, and the

${ }^{1-4}$ Consultant, ${ }^{5}$ Director

${ }^{1-5}$ Department of Orthopaedic Surgery, Division of Shoulder Surgery, The Johns Hopkins University, Baltimore, Maryland United States

Corresponding Author: Edward G McFarland, Director Department of Orthopaedic Surgery, Division of Shoulder Surgery, The Johns Hopkins University, Baltimore, Maryland United States, Phone: +4105832851, e-mail: emacfarl1@jhmi.edu incidence increases with age. ${ }^{1}$ The optimal treatment of these fractures in adults, especially those with osteoporotic bone, is debated. Depending on fracture type, treatment options are nonoperative treatment, percutaneous pin fixation, open reduction and internal fixation, HA, and RTSA. Factors that influence the choice of treatment are patient age, arm dominance, patient activity level, presence of other injuries, quality of bone, and configuration of the fracture (especially comminution, displacement, dislocation of the humeral head, head-splitting fracture, and medial column disruption). ${ }^{2}$

For patients in whom nonoperative treatment or open reduction and internal fixation is not optimal, HA has been the mainstay, producing satisfactory results. ${ }^{3-5} \mathrm{Neer}^{6}$ published one of the first studies of HA to treat proximal humerus fractures, reporting that 39 of 43 outcomes were good to excellent. Subsequent studies were unable to duplicate those results. ${ }^{7-9}$ Poor results because of tuberosity malunion, nonunion or resorption of the tuberosities, or rotator cuff tearing have been reported ${ }^{8,10}$ (Fig. 1). All of these mechanisms of failure produce a rotator cuffdeficient shoulder with subsequent shoulder weakness, loss of motion, and, in some cases, pain. ${ }^{10}$

Reverse total shoulder arthroplasty was designed for patients with cuff tear arthropathy or large rotator cuff tears and arthritis. The prosthetic design of the RTSA

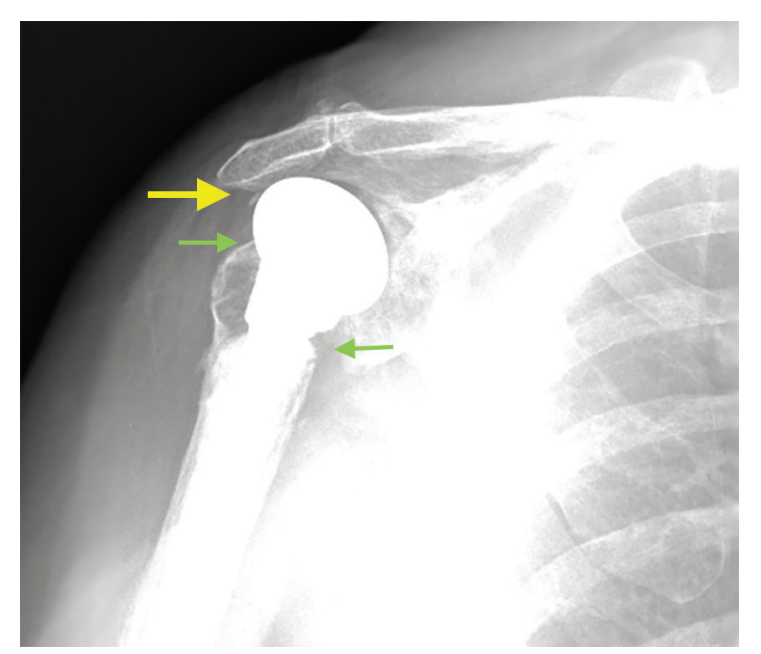

Fig. 1: Anteroposterior radiograph of a hemiarthroplasty placed for a comminuted proximal humerus fracture with subsequent superior subluxation and superior glenoid wear 

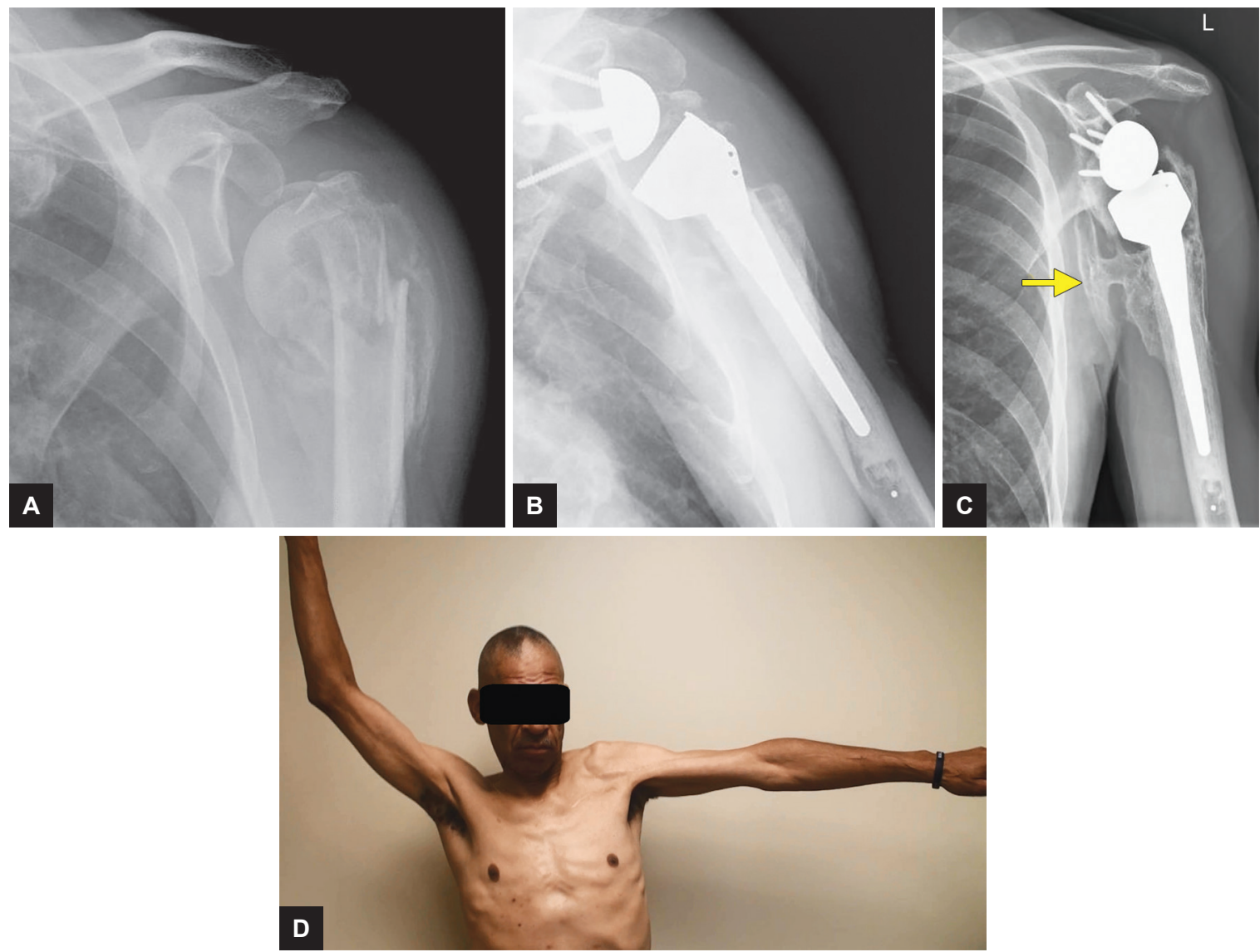

Figs 2A to D: (A) Anteroposterior radiograph of a proximal humerus fracture 6 weeks after revascularization of an arterial injury; (B) anteroposterior radiograph of RTSA taken 1 week postoperatively; (C) extreme heterotopic ossification 9 months after implantation of the RTSA; and (D) despite limited range of motion, patient was satisfied with his pain relief and ability to continue to work as a house painter

allows the deltoid muscle to elevate the arm without an intact rotator cuff. Because of the high incidence of rotator cuff dysfunction after HA for the treatment of proximal humerus fractures, RTSA has been used in patients with rotator cuff deficiency or those in whom tuberosity osteosynthesis is unachievable. ${ }^{11,12}$ Studies of RTSA for the treatment of patients with proximal humerus fractures have shown acceptable results clinically and radiographically ${ }^{13,14}$ (Fig. 2).

There is increasing controversy regarding whether HA or RTSA provides the best results for surgical treatment of proximal humerus fractures. The goal of this systematic review was to compare the clinical results of HA vs RTSA for the treatment of proximal humerus fractures.

\section{MATERIALS AND METHODS}

We searched the MEDLINE/PubMed, Excerpta Medica/ EMBASE, and Scopus databases for articles through January 2015 using the following terms, plus combinations of these terms: "Proximal humerus fracture," "shoulder hemiarthroplasty," "reverse shoulder arthroplasty," and "reverse total shoulder arthroplasty." We included articles with evidence level I, II, III, or IV, comparing outcomes of adults after RTSA or HA for acute and traumatic proximal humerus fractures. We also reviewed the bibliography of each article that met our inclusion criteria to identify studies that may have been missed by the original search. We applied the following exclusion criteria: Publication in a language other than English, animal/cadaveric/basic science studies, level V evidence, case reports, failure to report clinical outcomes, letters to the editor, meeting presentations, and studies about revision RTSA for failed primary procedures. The level of evidence for each study was determined by two of the authors (AB and EGM) using commonly accepted criteria. ${ }^{15}$ In the present review, only articles that met accepted quality standards for design and reporting were included.$^{16}$ No attempt was made to gather unpublished data or to retrieve additional information from any of the authors of the studies.

Our search produced 141 studies. Of these, eight compared RTSA with HA for the treatment of acute proximal humerus fractures. ${ }^{13,14,17-22}$ These eight studies reported 180 patients treated with RTSA and 439 patients treated with HA. One study was a prospective, blinded, randomized controlled trial (level I), ${ }_{1}^{14}$ and one was a prospective, 
nonrandomized study (level II). ${ }^{20}$ The remaining six studies were retrospective cohort studies (level III). All were analyzed for variables that are important in choosing treatment options. We evaluated each study for subjective, patient-reported measures [i.e., the ASES score, the Disabilities of the Arm, Shoulder, and Hand (DASH) scoring system, the absolute Constant-Murley, the modified Constant-Murley score, and the Simple Shoulder Test (SST)]. We compared objective measures of range of motion after surgery, and we evaluated radiographic results. For the studies that reported radiographic results, the Nerot-Sirveaux grading system ${ }^{23}$ was used when the studies graded scapular notching. Lastly, we compared complication rates between the two groups.

\section{Statistical Analysis}

Patient demographic data, range of motion, and outcome scores were gathered from each study, and frequencyweighted means were calculated. All outcomes were analyzed as continuous variables. Differences in final outcomes between the RTSA and HA groups were analyzed with an independent sample Student's t-test. Statistical analysis was performed using Statistical Package for the Social Sciences statistics, version 22.0 software (IBM Corp, Armonk, New York, USA). Reported mean values are frequency-weighted. Statistical significance was set at a $\mathrm{p}$-value of $<0.05$.

\section{RESULTS}

The mean age in the RTSA group (77 years) was significantly higher than in the HA group (72 years; $p=0.0001$; Table 1). There was no significant difference in the proportion of women in the RTSA group (85\%) compared with the HA group $(79 \% ; \mathrm{p}=0.50)$. There was no significant difference in the mean length of follow-up for the RTSA group (3.0 years) compared with the HA group (4.3 years; $p=0.18$ ). The predominant fracture patterns in both groups (93\% of cases) were three-part or four-part fractures with or without dislocation. The remainder were two-part fractures. A deltopectoral approach was used in six studies, ${ }^{14,17-20,22}$ and either a superolateral or deltopectoral approach was used in two studies. ${ }^{13,21}$ The greater and lesser tuberosities were excised in one study. ${ }^{13}$ The tuberosities were reattached in six studies. ${ }^{13,14,17,20-22}$ In two ${ }^{14,21}$ of those studies, a technique described by Boileau et $\mathrm{al}^{10}$ was used for tuberosity reconstruction. In this technique, heavy (no. 5) nonabsorbable sutures are used for tuberosity osteosynthesis to attach tuberosity fragments to the humeral shaft, to each other, and to the prosthesis. ${ }^{10}$

\section{Outcomes}

There was lack of uniformity among the studies regarding which clinical outcome scores were used. The ASES score was used in five studies, ${ }^{17,19-22}$ the DASH in three studies, ${ }^{13,14,18}$ the absolute Constant-Murley score in three studies, ${ }^{13,14,18}$ the modified Constant-Murley score in two studies, ${ }^{14,18}$ and the SST in two studies. ${ }^{19,20}$

Reverse total shoulder arthroplasty was more effective than HA in alleviating pain (Table 2). The mean ConstantMurley pain score was 14.5 in the RTSA group compared with 9.1 in the HA group $(p=0.004)$. The RTSA group had a higher mean ASES pain score of 52 compared with 43 in the HA group, but this difference was not statistically significant $(\mathrm{p}=0.5)$.

Patient-reported outcomes were better in the RTSA than in the HA group (Table 2). In the studies using the ASES score, the RTSA group reported a significantly better score (mean, 74) than the HA group (mean, 58; $\mathrm{p}=0.016$ ). In studies using absolute Constant-Murley score, RTSA patients showed a significantly better result (mean, 55) than the HA group (mean, 41; $\mathrm{p}=0.001$ ). In studies using the modified Constant-Murley score, the RTSA group showed a significantly better postoperative score (mean,

Table 1: Characteristics of patients with a proximal humerus fracture who underwent RTSA or HA for treatment

\begin{tabular}{|c|c|c|c|c|c|c|c|}
\hline \multirow[b]{2}{*}{ Study } & \multirow{2}{*}{$\begin{array}{l}\text { Level of } \\
\text { evidence }\end{array}$} & \multicolumn{2}{|c|}{ Mean age (range), $y$} & \multicolumn{2}{|c|}{ No. (\% Women) } & \multicolumn{2}{|c|}{ Mean (range) length of follow-up, $y$} \\
\hline & & RTSA & $H A$ & RTSA & $H A$ & RTSA & $H A$ \\
\hline Baudi ${ }^{17}$ & III & 77 & 71 & 25 (NR) & $28(\mathrm{NR})$ & 2.2 & 2.2 \\
\hline Boyle $^{18}$ & III & $80(57-90)$ & $72(27-96)$ & $55(93)$ & $313(78)$ & $5.0^{\ddagger}$ & $5.0^{\ddagger}$ \\
\hline Chalmers $^{19}$ & III & $77 \pm 6^{*}$ & $72 \pm 7^{*}$ & $9(78)$ & $9(78)$ & $1.2 \pm 0.5^{*}$ & $4.9 \pm 1.2^{*}$ \\
\hline Cuff 20 & II & $75(70-86)$ & $74(70-88)$ & $24(58)$ & $23(62)$ & $2.4(2.0-3.0)$ & $3.2(3.0-4.0)$ \\
\hline Gallinet $^{13}$ & III & $74(58-84)$ & $74(49-95)$ & $16(81)$ & $17(88)$ & $1.0(0.5-1.5)$ & $1.4(0.5-4.6)$ \\
\hline Garrigues $^{21}$ & III & $81(67-97)$ & $69(57-87)$ & $10(N R)$ & $9(\mathrm{NR})$ & $3.6(1.3-8)$ & \\
\hline Sebastiá-Forcada ${ }^{14}$ & I & $75(70-85)$ & $73(70-83)$ & $31(87)$ & $30(83)$ & $2.4(2.0-3.7)$ & $2.3(2.0-4.1)$ \\
\hline Young $^{22}$ & III & 77 & 75 & $10(100)$ & $10(80)$ & $1.8(1.3-3.1)$ & $3.7(2-4.7)$ \\
\hline FWM & & $77^{\dagger}$ & $72^{\dagger}$ & $180(85)^{\dagger}$ & $439(79)^{\dagger}$ & $3.0^{\dagger}$ & $4.3^{\dagger}$ \\
\hline$p$-value & & \multicolumn{2}{|c|}{0.0001} & \multicolumn{2}{|c|}{$0.50^{\S}$} & \multicolumn{2}{|c|}{0.18} \\
\hline
\end{tabular}

FWM: Frequency-weighted mean; NR: Not reported; *Expressed as mean \pm standard deviation; ${ }^{\dagger}$ Includes no data for the studies by Garrigues et al. and Baudi et al; ${ }^{\ddagger}$ All patients were followed for 5 years; $\S_{p}$-value for difference in proportion of women 
Table 2: Postoperative outcomes for patients with proximal humerus fracture treated with RTSA or HA

\begin{tabular}{|c|c|c|c|c|c|}
\hline \multirow[b]{2}{*}{ Outcome } & \multicolumn{2}{|c|}{ RTSA } & \multicolumn{2}{|c|}{$H A$} & \multirow[b]{2}{*}{$p$-value } \\
\hline & No. of patients & $F W M$ & No. of patients & $F W M$ & \\
\hline \multicolumn{6}{|l|}{ Pain relief } \\
\hline ASES pain score & 59 & 52 & 61 & 43 & 0.504 \\
\hline Constant-Murley pain score & 41 & 14.5 & 45 & 9.1 & 0.004 \\
\hline \multicolumn{6}{|l|}{ Patient-reported outcomes } \\
\hline ASES & 78 & 74 & 79 & 57 & 0.016 \\
\hline Constant-Murley (absolute) & 72 & 55 & 75 & 41 & 0.001 \\
\hline Constant Murley (modified) & 56 & 80.5 & 58 & 42 & 0.004 \\
\hline DASH & 72 & 30 & 75 & 28 & 0.60 \\
\hline SST & 33 & 7.3 & 32 & 6.1 & 0.33 \\
\hline \multicolumn{6}{|l|}{ Range of motion (degree) } \\
\hline Active abduction & 72 & 97 & 75 & 106.5 & 0.68 \\
\hline Active anterior elevation & 125 & 124 & 126 & 87 & 0.003 \\
\hline External rotation with arm by side & 65 & 17 & 68 & 21.5 & 0.47 \\
\hline External rotation with $90^{\circ}$ abduction & 19 & 45 & 19 & 38 & 0.582 \\
\hline Internal rotation with $90^{\circ}$ abduction & 35 & 36 & 35 & 46 & 0.39 \\
\hline
\end{tabular}

80.5) than the HA group (mean, 42; $\mathrm{p}=0.004)$. Although a better DASH score was observed in the RTSA group (mean, 30), it was not significantly better than that of the HA group (mean, 28; $\mathrm{p}=0.60$ ). Similarly, a better SST score was observed in the RTSA group (mean, 7.3) than in the HA group (mean, 6.1), although the difference was not significant $(p=0.33)$.

The proximal humerus fracture prevented patients from moving their arms before surgery. Therefore, postoperative range of motion (and not improvement in range of motion) was evaluated (Table 2). The RTSA group showed significantly better mean postoperative active anterior elevation $\left(124^{\circ}\right)$ compared with the HA group $\left(87^{\circ} ; \mathrm{p}=0.003\right)$. Although the HA group had higher mean active abduction $\left(106.5^{\circ}\right)$ than the RTSA group $\left(97^{\circ}\right)$ and greater external rotation with the arm by the side $\left(21.5^{\circ}\right.$ vs $17^{\circ}$ ), these differences were not statistically significant $(\mathrm{p}=0.68$ and 0.47 respectively). Only one study evaluated the effect of tuberosity healing on final range of motion, ${ }^{20}$ reporting that those with healing of the tuberosities on radiographs $(n=14)$ had better external rotation $\left(28^{\circ}\right)$ than those with resorption of the tuberosities $(n=9$; external rotation, $5^{\circ} ; \mathrm{p}=0.009$ ).

The overall complication rates between the RTSA group (21 complications; $11.8 \%$ ) and the HA group (38 complications; $20.5 \%$ ) were not significantly different $(p=0.36$; Table 3$)$. Stiffness was the only complication that differed significantly between groups, with rates of $4.4 \%$ in the HA group and $0 \%$ in the RTSA group $(p=0.03)$. The rate of heterotopic ossification was $2 \%$ in the RTSA group compared with $3.8 \%$ in the HA group $(\mathrm{p}=0.36)$. The RTSA group showed scapular notching in $15.8 \%$ of cases. Of the cases with scapular notching, 53\% were
Table 3: Complication type as percent of total complications after RTSA or HA for treatment of proximal humerus fracture

\begin{tabular}{llll}
\hline \multirow{2}{*}{ Complication } & \multicolumn{3}{c}{$\begin{array}{c}\text { No. (\%) of } \\
\text { complications }\end{array}$} \\
\cline { 2 - 3 } Dislocation & $R T S A$ & $H A$ & \\
Hematoma & 0 & $6(2.5)$ & 0.33 \\
Infection & $1(0.4)$ & $1(0.5)$ & 0.90 \\
Malunion & $3(2)$ & $4(2)$ & 0.97 \\
Neural complications & $6(2.4)$ & $4(1.7)$ & 0.8 \\
Nonunion & $2(1)$ & $3(2.8)$ & 0.42 \\
Periprosthetic fracture & $1(1.3)$ & 0 & 0.33 \\
Pulmonary complications & $1(0.5)$ & $1(0.4)$ & 0.87 \\
Reflex sympathetic dystrophy & 0 & $2(0.9)$ & 0.14 \\
Resorption & $2(2.2)$ & $2(1.5)$ & 0.74 \\
Stiffness & $5(2)$ & $9(3.8)$ & 0.69 \\
Overall & 0 & $6(4.4)$ & 0.03 \\
\hline
\end{tabular}

grade I, $32 \%$ were grade II, $16 \%$ were grade III, and $0 \%$ were grade IV.

\section{DISCUSSION}

This review shows that, within the limits of the available evidence, the use of RTSA in acute proximal humerus fractures results in statistically better pain relief, range of motion in elevation, and ASES and Constant-Murley scores compared with that of HA. We speculate that the reason RTSA provides better results is that it does not depend on an intact rotator cuff for pain relief and improvement in motion.

Studies have suggested that the primary factor associated with patient satisfaction after shoulder arthroplasty 
is pain relief. ${ }^{24,25}$ For patients who undergo arthroplasty for complex proximal humerus fractures, RTSA provides significantly better pain relief than HA. Patients with HA frequently comment that they do not have pain until they move their arms, ${ }^{26,27}$ whereas RTSA patients do not experience pain from superior subluxation or contact between the metal prosthesis and the bone of the glenoid. Patients who have undergone RTSA also report less pain with activities of daily living, which is shown in the significantly lower Constant-Murley and ASES pain scores of the RTSA group compared with those of the HA group.

Although the two patient groups studied here were fairly similar, undetectable differences may have influenced the results. For example, proximal humerus fractures are a complex group of fractures that can include two-part fractures or complex fracture dislocations of the shoulder. The Neer classification system of fracture patterns was used in all studies, and it has been shown to have poor interrater reliability. ${ }^{28,29}$ There were insufficient data reported by each study to determine the exact distribution of proximal humerus fracture types. It is important to note that the results of this study do not relate to the results of HA or RTSA for surgical neck fractures, isolated greater tuberosity fractures, or proximal humerus fracture malunions. The time-to-surgery might also have affected the results, but most studies did not report this variable.

Postoperative range of motion, particularly active anterior elevation, was significantly better in the RTSA group compared with the HA group. This is likely because of better pain relief and function after RTSA. Only one study evaluated the importance of healing of the greater tuberosity on external rotation after RTSA. ${ }^{20}$ Neither of the prostheses implanted during surgery in the studies included here resulted in normal shoulder range of motion. The limited range of shoulder motion after both procedures may indicate soft tissue damage and subsequent scarring. It is important for patients and treating physicians to understand that normal range of motion after HA or RTSA for complex proximal humerus fractures is unlikely.

Patient-reported outcome measures showed significantly better Constant-Murley and ASES scores in the RTSA group compared with those of the HA group. Although significantly different, these differences may not be clinically meaningful. The minimal clinically important difference for the ASES score from preoperative values to postoperative values for arthroplasty has been reported to be 6.4 points. ${ }^{30}$ No preoperative values were available in the studies reported here because the tests could not be performed preoperatively in patients with shoulder fractures. Also, because absolute values defining "good" scores have not been determined for the clinical outcome measures assessed here, the clinical superiority of one type of score $v s$ another is unknown. Another limitation is the lack of uniformity of measurement instruments across studies. Patient-reported outcome scores may be less accurate in this population because they were validated in patients with osteoarthritis and not proximal humerus fractures.

The complications reported in these studies highlight important differences between the use of RTSA and HA for complex proximal humerus fractures. Some complications, such as dislocation, were not present in the RTSA group. The dislocation rate in patients with proximal humerus fractures treated with RTSA differs substantially from that in patients with cuff tear arthropathy treated with RTSA, which has been reported to be as high as $31 \% .{ }^{31}$ Rates of complications common to most shoulder arthroplasties, such as infection and nerve damage, were not different between the two groups. Some complications, such as base-plate loosening, were seen only in the RTSA group and may occur during longer follow-up than was available in these studies.

The main strength of this systematic review is that the numbers obtained are greater than could be provided by any one center or practitioner. This allowed statistical analysis of variables of great importance to patients and surgeons providing treatment, and it allows future studies to be compared against the results obtained here. Under the quality rating criteria for systematic reviews, ${ }^{32}$ this review would rank at the level of "good."

Several limitations of our study are inherent to systematic reviews. Most studies (6 of 8 ) were retrospective, and there was only one randomized prospective study. This lack of randomization and blinding introduces a higher likelihood of bias. Because of the small number of included studies, this review reflects outcomes of a particular population of surgeons. Results and complication rates may be different with surgeons of different experience levels. Similarly, complication rates may be higher with longer follow-up. Also, comparability of range of motion data can be compromised by inconsistent measurement techniques, differences in measuring devices, and differences in whether active or passive motion was recorded. This study did not evaluate the cost of RTSA, $\mathrm{HA}$, or perioperative care and rehabilitation. It is possible that one procedure is more cost-effective given the modest differences in clinical results.

In conclusion, using the available literature, this systematic review found that for complex proximal humerus fractures, RTSA resulted in better pain relief, similar range of motion, better ASES and Constant-Murley scores, and similar complication rates when compared with HA. Although the differences in the results between the two procedures were statistically significant, further study is warranted to determine whether they are clinically important and if RTSA is cost-effective. 


\section{REFERENCES}

1. Court-Brown CM, Caesar B. Epidemiology of adult fractures: a review. Injury 2006 Aug;37(8):691-697.

2. Hageman MG, JayakumarP,KingJD, Guitton TG, DoornbergJN, Ring D. The factors influencing the decision making of operative treatment for proximal humeral fractures. J Shoulder Elbow Surg 2015 Jan;24(1):e21-e26.

3. Goldman RT, KovalKJ, CuomoF, GallagherMA,ZuckermanJD. Functional outcome after humeral head replacement for acute three- and four-part proximal humeral fractures. J Shoulder Elbow Surg 1995 Mar-Apr;4(2):81-86.

4. Levine WN, Connor PM, Yamaguchi K, Self EB, Arroyo JS, Pollock RG, Flatow EL, Bigliani LU. Humeral head replacement for proximal humeral fractures. Orthopedics 1998 Jan;21(1):68-73; quiz 74-75.

5. Zyto K, Wallace WA, Frostick SP, Preston BJ. Outcome after hemiarthroplasty for three- and four-part fractures of the proximal humerus. J Shoulder Elbow Surg 1998 MarApr;7(2):85-89.

6. Neer CS II. Displaced proximal humeral fractures. II. Treatment of three-part and four-part displacement. J Bone Joint Surg Am 1970 Sep;52(6):1090-1103.

7. Besch L, Daniels-Wredenhagen M, Mueller M, Varoga D, Hilgert RE, Seekamp A. Hemiarthroplasty of the shoulder after four-part fracture of the humeral head: a long-term analysis of 34 cases. J Trauma 2009 Jan;66(1):211-214.

8. Kontakis G, Koutras C, Tosounidis T, Giannoudis P. Early management of proximal humeral fractures with hemiarthroplasty: a systematic review. J Bone Joint Surg Br 2008 Nov;90(11):1407-1413.

9. Robinson CM, Page RS, Hill RM, Sanders DL, Court-Brown CM, Wakefield AE. Primary hemiarthroplasty for treatment of proximal humeral fractures. J Bone Joint Surg Am 2003 Jul; 85-A(7):1215-1223.

10. Boileau P, Krishnan SG, Tinsi L, Walch G, Coste JS, Mole D. Tuberosity malposition and migration: reasons for poor outcomes after hemiarthroplasty for displaced fractures of the proximal humerus. J Shoulder Elbow Surg 2002 Sep-Oct; 11(5):401-412.

11. Gerber C, Pennington SD, Nyffeler RW. Reverse total shoulder arthroplasty. J Am Acad Orthop Surg 2009 May;17(5):284-295.

12. Lenarz C, Shishani Y, McCrum C, Nowinski RJ, Edwards TB, Gobezie R. Is reverse shoulder arthroplasty appropriate for the treatment of fractures in the older patient? Early observations. Clin Orthop Relat Res 2011 Dec;469(12):3324-3331.

13. Gallinet D, Clappaz P, Garbuio P, Tropet Y, Obert L. Three or four parts complex proximal humerus fractures: hemiarthroplasty versus reverse prosthesis: a comparative study of 40 cases. Orthop Traumatol Surg Res 2009 Feb;95(1):48-55.

14. Sebastia-Forcada E, Cebrian-Gomez R, Lizaur-Utrilla A, Gil-Guillen V. Reverse shoulder arthroplasty versus hemiarthroplasty for acute proximal humeral fractures. A blinded, randomized, controlled, prospective study. J Shoulder Elbow Surg 2014 Oct;23(10):1419-1426.

15. Wright RW, Kuhn JE, Amendola A, Jones MH, Spindler KP. Symposium integrating evidence-based medicine into clinical practice. J Bone Joint Surg Am 2007 Jan;89(1):199-205.

16. Gasparyan AY, Ayvazyan L, Blackmore H, Kitas GD. Writing a narrative biomedical review: considerations for authors, peer reviewers, and editors. Rheumatol Int 2011 Nov;31(11):1409-1417.

17. Baudi P, Campochiaro G, Serafini F, Gazzotti G, Matino G, Rovesta C, Catani F. Hemiarthroplasty versus reverse shoulder arthroplasty: comparative study of functional and radiological outcomes in the treatment of acute proximal humerus fracture. Musculoskelet Surg 2014 Apr;98 (Suppl 1):S19-S25.

18. Boyle MJ, Youn SM, Frampton CM, Ball CM. Functional outcomes of reverse shoulder arthroplasty compared with hemiarthroplasty for acute proximal humeral fractures. J Shoulder Elbow Surg 2013 Jan;22(1):32-37.

19. Chalmers PN, Slikker W III, Mall NA, Gupta AK, Rahman Z, Enriquez D, Nicholson GP. Reverse total shoulder arthroplasty for acute proximal humeral fracture: comparison to open reduction-internal fixation and hemiarthroplasty. J Shoulder Elbow Surg 2014 Feb;23(2):197-204.

20. Cuff DJ, Pupello DR. Comparison of hemiarthroplasty and reverse shoulder arthroplasty for the treatment of proximal humeral fractures in elderly patients. J Bone Joint Surg Am 2013 Nov 20;95(22):2050-2055.

21. Garrigues GE, Johnston PS, Pepe MD, Tucker BS, Ramsey ML, Austin LS. Hemiarthroplasty versus reverse total shoulder arthroplasty for acute proximal humerus fractures in elderly patients. Orthopedics 2012 May;35(5):e703-e708.

22. Young SW, Segal BS, Turner PC, Poon PC. Comparison of functional outcomes of reverse shoulder arthroplasty versus hemiarthroplasty in the primary treatment of acute proximal humerus fracture. ANZ J Surg 2010 Nov;80(11):789-793.

23. Sirveaux F, Favard L, Oudet D, Huquet D, Walch G, Mole D. Grammont inverted total shoulder arthroplasty in the treatment of glenohumeral osteoarthritis with massive rupture of the cuff. Results of a multicentre study of 80 shoulders. J Bone Joint Surg Br 2004 Apr;86(3):388-395.

24. Chen AL, Bain EB, Horan MP, Hawkins RJ. Determinants of patient satisfaction with outcome after shoulder arthroplasty. J Shoulder Elbow Surg 2007 Jan-Feb;16(1):25-30.

25. Harreld K, Clark R, Downes K, Virani N, Frankle M. Correlation of subjective and objective measures before and after shoulder arthroplasty. Orthopedics 2013 Jun;36(6):808-814.

26. Mighell MA, Kolm GP, Collinge CA, Frankle MA. Outcomes of hemiarthroplasty for fractures of the proximal humerus. J Shoulder Elbow Surg 2003 Nov-Dec;12(6):569-577.

27. Moeckel BH, Dines DM, Warren RF, Altchek DW. Modular hemiarthroplasty for fractures of the proximal part of the humerus. J Bone Joint Surg Am 1992 Jul;74(6):884-889.

28. Bernstein J, Adler LM, Blank JE, Dalsey RM, Williams GR, Iannotti JP. Evaluation of the Neer system of classification of proximal humeral fractures with computerized tomographic scans and plain radiographs. J Bone Joint Surg Am 1996 Sep;78(9):1371-1375.

29. Foroohar A, Tosti R, Richmond JM, Gaughan JP, Ilyas AM. Classification and treatment of proximal humerus fractures: inter-observer reliability and agreement across imaging modalities and experience. J Orthop Surg Res 2011 Jul 29;6:38.

30. Michener LA, McClure PW, Sennett BJ. American Shoulder and Elbow Surgeons Standardized Shoulder Assessment Form, patient self-report section: reliability, validity, and responsiveness. J Shoulder Elbow Surg 2002 Nov-Dec;11(6):587-594.

31. Cheung E, Willis M, Walker M, Clark R, Frankle MA. Complications in reverse total shoulder arthroplasty. J Am Acad Orthop Surg 2011 Jul;19(7):439-449.

32. Harris RP, Helfand M, Woolf SH, Lohr KN, Mulrow CD, Teutsch SM, Atkins D, Methods Work Group, Third US Preventive Services Task Force. Current methods of the U.S. Preventive Services Task Force: a review of the process. Am J Prev Med 2001 Apr;20(3 Suppl):21-35. 